\title{
Research on joint optimal scheduling of air conditioning load and electric vehicle under demand response
}

\author{
Haiyu Huang ${ }^{1}$, Chunming Wang ${ }^{1}$, Shaolian Xia ${ }^{1}$, Huaqiang Xiong ${ }^{1}$, Baofeng Jiang ${ }^{1}$ Dunnan Liu ${ }^{2}$ and GaoYuan ${ }^{2 *}$ \\ ${ }^{1}$ Central China Electric Power Dispatching and Control Sub-center of State Grid, Wuhan 430061, China \\ ${ }^{2}$ State Key Laboratory of Alternate Electrical Power System With Renewable Energy Sources(North China Electric Power University), \\ Changping District, Beijing 102206, China
}

\begin{abstract}
As an important part of energy Internet carrier, demand side resources can participate in many interactions with power grid. In order to reduce the peak to valley load difference of power grid, from the perspective of tapping the combined peak shaving potential of air conditioning load and electric vehicles, guided by TOU price and direct load control, this paper proposes an optimal scheduling model with the minimum load difference and the maximum total revenue of users as the objective function. The results show that the joint optimal scheduling strategy can reduce the peak load and eliminate the "secondary peak load" caused by disorderly charging of electric vehicles.
\end{abstract}

\section{Introduction}

Electric vehicles have typical dual attributes of load and power supply, which play an important role in the construction of safe, economic and environmental protection intelligent power system, and are also one of the important means to solve the traffic, energy and environmental problems [1-2]. As the most important part of heating load and cooling load in winter and summer, air conditioning load plays a dominant role in temperature control load. At present, electric vehicles and air conditioning load account for a large proportion of household and commercial power consumption, so it is feasible and necessary to study the demand side response of both.

Air conditioning load and electric vehicle are typical controllable load. There are many researches on the controllable load participating in power system operation by scholars at home and abroad. Literature [4] uses the characteristics of different energy storage forms to improve the volatility of wind power. In reference [5], a hierarchical optimal scheduling scheme for thermal power units with energy storage assisted deep peak shaving is proposed. Literature [6] proposed the aggregation modeling method of air conditioning load based on state transition theory, and literature [7] analyzed the energy consumption characteristics of air conditioning load, and took residents' air conditioning load as demand response resources to participate in demand response projects. In reference [8], the problem of temperature control load participating in system load balance in hour scale was studied. Based on the energy storage modeling of electric vehicles, the dynamic demand response model of electric vehicles participating in auxiliary peak shaving was established in reference
[9]. Reference [10] makes full use of the energy storage characteristics of electric vehicles, controls the operation mode of electric vehicles, and realizes peak load cutting and valley filling of the system and suppresses load fluctuation through charging and discharging optimization. In reference [11], a direct load control method for optimal load distribution of HVAC is proposed.

Most of the above literatures focus on the modeling and operation regulation of air-conditioning load and electric vehicle controllable load, and seldom on the joint peak shaving potential of demand side resources. In order to reduce the peak to valley load difference, this paper proposes a joint optimal scheduling strategy of aggregated air conditioning load and electric vehicle. The cost model and user benefit model of power grid dispatching are established, and the corresponding constraints and objective functions are determined. The example shows that under the guidance of demand response, the joint optimal dispatch of the two can effectively realize the "peak load cutting and valley filling" of power system load, and ensure the revenue of users.

\section{Air conditioning load and electric vehicle cost under demand side dispatch}

\subsection{Air conditioning load dispatch cost}

For air-conditioning load, because of its high dispatching frequency, periodic start-up and shut-down has little impact on the unit life; the cost of user participation in regulation is low, which can be used as the base load of

* Corresponding author: gaoyuanhd@163.com 
peak load regulation, and low electricity price compensation method can be adopted, that is, fixed electricity price subsidy is carried out according to the regulation capacity during operation, but no compensation is given in case of interruption. The scheduling cost is as follows.

$$
C_{\mathrm{al}}=\sum_{i=1}^{N} \sum_{j=1}^{n_{A C}} \gamma_{0} \mathrm{u}_{A C}(i, j) P_{A C}(i, j) \Delta t
$$

Among them, $\gamma_{0}$ is the compensation rate of electricity price, which is taken as 0.2 in this paper; $\mathrm{u}_{A C}(i, j)$ is the state decision variable of the $\mathrm{jth}$ air conditioning unit in I period, where 0 is on, 1 is off, and $P_{A C}(i, j)$ is the power of the jth air conditioning unit in I period; $\Delta t$ is the interval of dispatching period, which is taken as 15 minutes in this paper; $n$ is the total number of dispatching periods, and $n_{A C}$ is the number of air conditioning units.

\subsection{Air conditioning load dispatch cost}

For electric vehicles, they are often used as commuting tools. The owners of electric vehicles have strict energy consumption plan and high cost of battery loss. In order to stimulate the enthusiasm of users, high price compensation can be used, that is, discount price is used when EV is charged by dispatching, and high price compensation is used when EV is discharged.

(1) Electric vehicle charging compensation

$$
C_{\mathrm{ec}}=\sum_{i=1}^{N} \sum_{j=1}^{n_{E V}}(1-\gamma) P_{r} \mathrm{u}_{E V C}(i, j) \eta_{c} P_{E V C}(i, j) \Delta t
$$

Among them, $n_{E V}$ is the number of electric vehicles; $\gamma$ is the discount rate of electricity price, which is 0.8 in this paper; $P_{r}$ is the real-time electricity price; $\mathrm{u}_{E V C}(i, j)$ is the state variable, 1 is charging, 0 is neither charging nor discharging; $P_{E V_{C}}(i, j)$ is the charging power of electric vehicle $\mathrm{j}$ in $\mathrm{i}$ period $(\mathrm{kw}) ; \eta_{c}$ is the charging efficiency of electric vehicle (dimensionless).

(2) Battery loss cost of electric vehicle

$$
C_{\mathrm{dch}}=\frac{P_{E V b}}{N_{E V b} Q_{E b}} Q_{D I S b}
$$

Among them, $P_{E V b}$ is the price of electric vehicle battery; $N_{E V b}$ is the number of charging and discharging cycles of electric vehicle battery during its service life; $Q_{E b}$ and $Q_{D I S b}$ are the rated and actual discharging capacity of battery respectively.

(3) Electric vehicle discharge compensation cost

$$
C_{E V \mathrm{~d}}=\sum_{i=1}^{N} \sum_{j=1}^{n_{E V}} P_{r} \mathrm{u}_{E V d}(i, j) \frac{1}{\eta_{d}} P_{E V d}(i, j) \Delta t
$$

Among them, $\mathrm{u}_{E V d}(i, j)$ is the state variable, 1 is discharge, 0 is no discharge; $P_{E V d}(i, j)$ is the actual discharge power $(\mathrm{kw})$ of electric vehicle $\mathrm{j}$ in $\mathrm{i}$ period, and $\eta_{d}$ is the discharge efficiency (dimensionless) of electric vehicle.

(4) Charge for disorderly charging of electric vehicles

$$
C_{\mathrm{cs}}=\sum_{i=1}^{N} \sum_{j=1}^{n_{E V}} P_{r} P_{o c}(i, j) \Delta t
$$

Among them, $P_{o c}(i, j)$ is the charging power of electric vehicle $\mathrm{j}$ in $\mathrm{i}$ period before dispatching.

(5) Cost of electric vehicle V2B

$$
C_{V 2 B}=\sum_{i=1}^{N} \sum_{j=1}^{n_{E V}}\left(P_{r} \mathrm{u}_{E V C}(i, j) P_{E V C}(i, j) \Delta t\right)-C_{\mathrm{ed}}+C_{d c h}
$$

\section{Joint optimal dispatch of temperature controlled load and electric vehicle}

\subsection{Objective function}

To apply aggregate air conditioning load and electric vehicle as peak shaving resources to load management, it is necessary to balance the interests of users and power grid to achieve win-win results.

(1)If the original daily load of power grid is $L_{n, i}(i,=1,2, \cdots, N)$, the charging load is $L_{E V c, i}$, the discharging load is $L_{E V d, i}$ and the aggregate power of air conditioning load is $P_{\text {agg, } i}$ in the period I after the joint optimal dispatch, then the grid load $L_{i}$ after the joint optimal dispatch is:

$$
L_{i}=L_{n, i}+L_{E V c, i}-L_{E V d, i}+P_{\mathrm{agg}, i}
$$

Considering from the power grid side, the main starting point is to realize "peak shaving and valley filling and smooth load curve". Therefore, the objective function is to minimize the load peak valley difference in the regulation period.

$$
\min F_{1}=\frac{\left[\max \left(L_{i}\right)-\min \left(L_{i}\right)\right]}{\max \left(L_{i}\right)}
$$

Among them, $\max (\mathrm{Li})$ is the peak load in the regulation period, and $\min (\mathrm{Li})$ is the valley load in the regulation period.

(2) The benefit of electric vehicle participating in joint optimal dispatching is the difference of power consumption cost between disorderly charging mode and V2b mode, while the benefit of air conditioning load is the subsidy cost of grid capacity, which is: the power consumption cost of the two modes is the difference of power consumption cost between disorderly charging mode and V2b mode.

$$
\left\{\begin{array}{l}
I_{E V}=C_{\mathrm{cs}}-C_{V 2 B} \\
I_{A C}=C_{a l}
\end{array}\right.
$$


Considering from the user side, we hope that the higher the revenue, the better, and take the total revenue of users as its objective function.

$$
\max I_{\text {total }}=\max \left(I_{A C}+I_{E V}\right)
$$

\subsection{Constraints}

(1) Air conditioning load start stop time constraint

Air conditioning load and indoor air have certain heat storage characteristics, so the total energy storage in the former period is larger than that in the latter period

The time relationship is as follows:

$$
\tau_{i}^{o f f}(t) \leq \tau_{o f f}
$$

$\tau_{\mathrm{i}}^{\text {off }}(t)$ is the initial state.

(2) Battery capacity constraints of electric vehicles

$$
S O C_{\min } \leq S O C(t) \leq S O C_{\max }
$$

Among them, $S O C_{\min }$ and $S O C_{\max }$ are the minimum and maximum battery capacity of electric vehicles respectively. Considering the service life of batteries, the values in this paper are 0.2 and 0.9 .

(3) Time constraint of charge and discharge

In order to minimize the cost of electric vehicles in the process of $\mathrm{V} 2 \mathrm{~B}$, the charging period should be selected in the period of low electricity price as far as possible, and the discharge period should be selected in the period of peak electricity price as far as possible:

$$
\left\{\begin{array}{l}
\mathrm{t}_{c} \in T_{e L} \\
\mathrm{t}_{d} \in T_{e H}
\end{array}\right.
$$

Among them, ${ }^{T_{e L}}$ and $T_{e H}$ are the periods of low price and high price under tou mode respectively.

(4) Charge discharge state constraint

The working state of electric vehicle at any moment is different, that is, it can only be in one of the two states of charging and discharging:

$$
\mathrm{u}_{E V C}(i, j) \cdot \mathrm{u}_{E V S}(i, j)=0
$$

\subsection{Model solving}

The joint optimal scheduling model is a multi-objective optimal solution problem, which is solved by the improved non dominated sorting genetic algorithm.

In the process of multi-objective problem solving, the solution is easy to fall into the local optimal solution, which can be expressed as a solution set, called non dominated solution or Pareto solution. In this chapter, the two optimized objectives need to be minimized, so the partial small fuzzy membership function in fuzzy theory is used to express them.

$$
\omega_{\mathrm{n}}=\left\{\begin{array}{cc}
1 & \mathrm{~F}_{n} \leq F_{n}^{\min } \\
\frac{F_{n}^{\max }-\mathrm{F}_{n}}{F_{n}^{\max }-F_{n}^{\min }} & F_{n}^{\max }>\mathrm{F}_{n}>F_{n}^{\min } \\
0 & \mathrm{~F}_{n} \geq F_{n}^{\max } \\
\omega=\frac{1}{m} \sum_{n=1}^{m} \omega_{\mathrm{n}}
\end{array}\right.
$$

Where $\omega_{\mathrm{n}}$ is 0 , it means that it is not satisfied with the nth objective function; $\omega_{\mathrm{n}}$ is 1 , it means that it is satisfied with the nth objective function; $F_{n}^{\max }$ is the maximum value of the nth objective function; $F_{n}^{\text {min }}$ is the minimum value of the nth objective function.

On the basis of the standardized satisfaction degree of the solution in the standardized Pareto solution obtained by equation 14, the Pareto optimal solution with the maximum value is selected as the optimal compromise solution by comparing with equation 15 .

\section{Example analysis}

The typical daily load curve of an office center in summer is shown in Figure 1. Among them, 180 air conditioners can be dispatched, the rated power of each air conditioner is 2 . Skw, and the energy efficiency ratio is 2.7. There are 50 electric vehicles, and the parameters are shown in Table 1. Taking time of use price as guidance price, 8:00-12:00, 16:00-20:00 are peak time price, 12:00-16:00, 20:00-24:00 are ordinary time price, 0:00-8:00 are valley time price. Considering the particularity of power consumption in office center, the regulation period is set as 8:00-18:00.

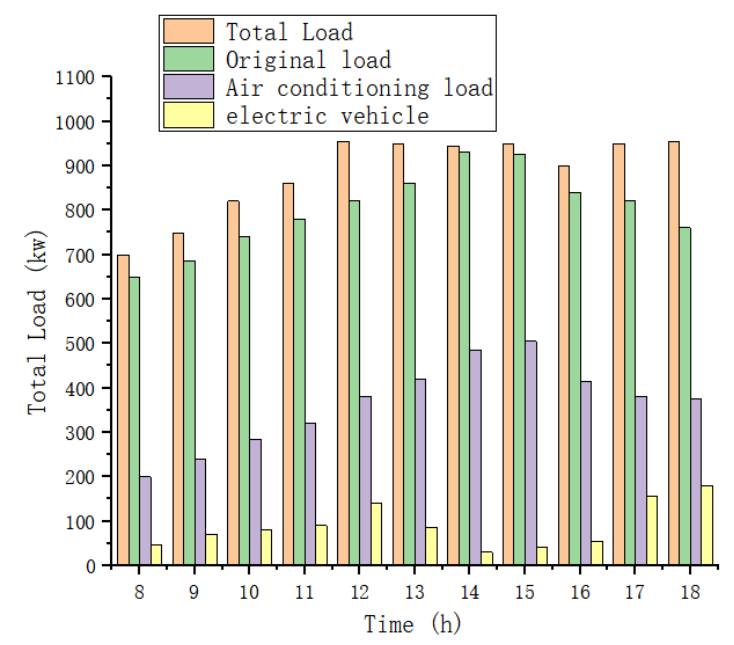

Fig. 1. Load distribution curve of office center

Table 1. Font styles for a reference to a journal article.

\begin{tabular}{cc}
\hline Parameter & Value \\
\hline Battery capacity (KWH) & 58.5 \\
Battery price (10000 yuan) & 6 \\
Charging times & 6000 \\
Rated charge discharge power (kw) & 9.6 \\
Charge discharge efficiency (\%) & 90 \\
\hline
\end{tabular}

First, analyze the air conditioning load or electric vehicle dispatching separately, and the load curve before and after dispatching is shown in Figure 2. It can be seen from Figure 2 that when the air conditioning load is separately dispatched, the load difference is $22.58 \%$, and the dispatch cost is 121.2 yuan. However, during the 
peak load period, the maximum power reduction of air conditioning load is $154 \mathrm{kw}$, which is $45.29 \%$ of the actual maximum regulating capacity of $340 \mathrm{kw}$, and the peak shaving margin of air conditioning load is not fully utilized; the reduction of "secondary peak load" is low, and the peak shaving effect is not obvious.

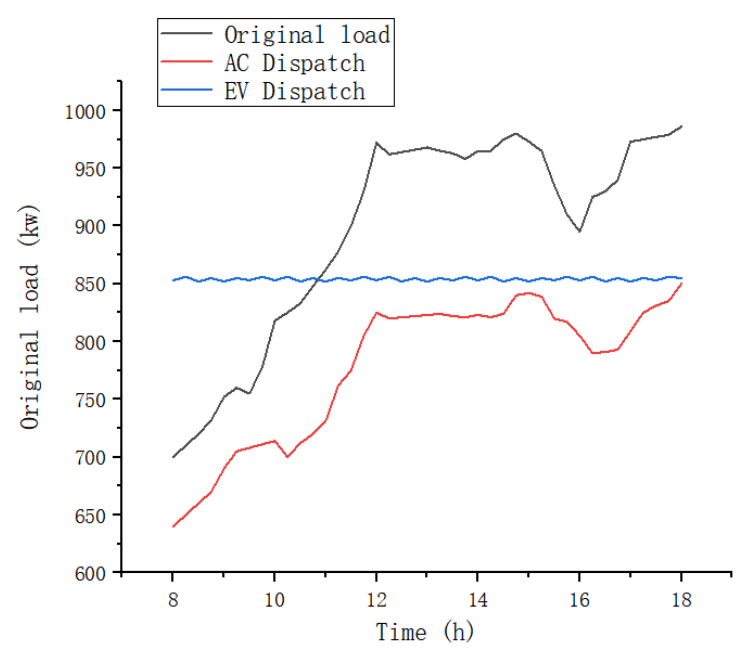

Fig. 2. Load curve of single dispatch

When the electric vehicle charging and discharging dispatching is carried out separately, a relatively constant power consumption can be maintained, the load difference is close to 0 , and the dispatching cost is 787.2 yuan; however, the centralized charging of electric vehicles must be carried out during the peak electricity price period, and the centralized discharging must be carried out during the peak load period (the average electricity price period), and the "secondary peak load" can only be reduced by reducing the charging amount. That is to say, charging in the period of high electricity price and discharging in the period of low electricity price lead to higher cost and lower economic feasibility for users to participate in V2b.

When the joint optimal dispatching strategy is adopted, the load curve before and after dispatching is shown in Figure 3.

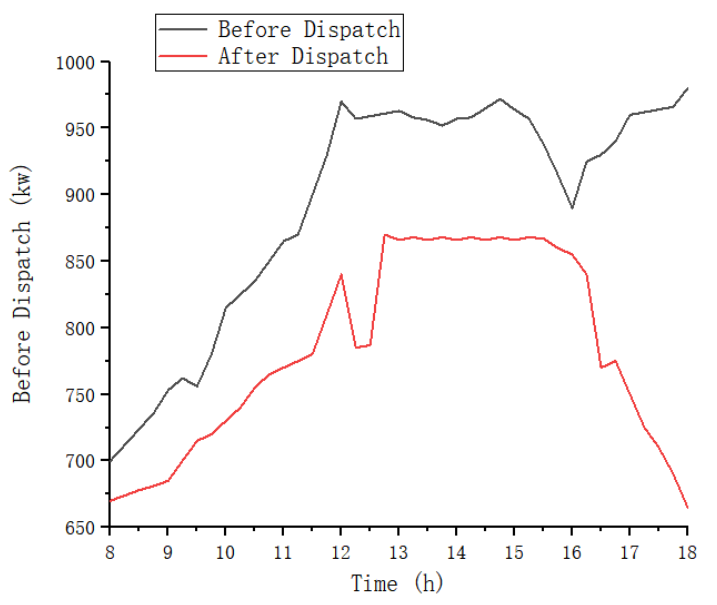

Fig. 3. Load curve before and after joint optimal dispatch

Under the joint optimal scheduling strategy, the air conditioning load regulation period is $12: 00-16: 00$; the electric vehicle charging period is 12:30-15:30; the discharge period is 16:30-18:00. At this time, the load difference is $22.22 \%$, and the dispatching cost is 722.7 yuan, including 160 yuan for air conditioning load compensation, 158.4 yuan for electric vehicle charging compensation, 404.3 yuan for electric vehicle discharging compensation, and 130.1 yuan for electric vehicle battery loss. The cost of electric vehicle participating in V2b is 359.4 yuan, the total cost of disorderly charging is 672.2 yuan, and the total revenue of users is 472.8 yuan. Under the premise of the same battery capacity change of a single electric vehicle, the charge for disorderly charging is 19.2 yuan; under the $\mathrm{V} 2 \mathrm{~b}$ mode, the charge is 12.0 yuan, and the cost is reduced by 7.2 yuan. It can be seen that the joint optimal scheduling mode is not only more conducive to load reduction than the single scheduling mode, but also can reduce the scheduling cost and improve the user income.

At the same time, the time of air-conditioning load regulation will be shortened by using the joint optimal dispatch (the whole time regulation of air-conditioning load will be carried out by dispatching time and space separately), which reduces the impact on the user's energy consumption comfort; the centralized charging of electric vehicles in the air-conditioning load regulation period realizes the complementary energy consumption and reduces the peak load; The effective discharge of electric vehicle users before work can eliminate the "second peak" of load.

\section{Conclusion}

In order to reduce the peak to valley load difference, from the perspective of tapping the combined peak shaving potential of air conditioning load and electric vehicles, guided by TOU price and direct load control, this paper proposes an optimal scheduling model with the minimum load difference and the maximum total revenue of users as the objective function. The results show that the joint optimal scheduling strategy can reduce the peak load and eliminate the "secondary peak load" caused by disorderly charging of electric vehicles.

\section{Acknowledgments}

This work was supported in part by National Natural Science Foundation of China under Grant 72001078 and Power System State Key Laboratory under Grant SKLD20M12.

\section{References}

1. Liu Dunnan, Wang Meibao, Jiang Yefeng, et al. Design of rapid demand response market mechanism based on load quality grading utilization .J. Journal of Global Energy Interconnection, (2019)

2. Xiao Jun, Yuan Weideng, Chen Jiahui, et al. Dynamic evaluation of power supply capacity considering demand response and network 
reconfiguration .J. Electrical Measurement and Instrumentation, (2019).

3. Wang Haojing, Wang Yufei, Zhang Yu, et a1.The orderly charging and discharging strategies of electric vehicles in the mode of V2G .J. Journal of Shanghai University of Electric Power, (2019).

4. Kempton W, Letendre S. Electric vehicles as a new power source for electric utilities .J.Transportation Research Part D23, (1997).

5. Callaway D S.Tapping the energy storage potential in electric loads to deliver load following and regulation, with application to wind energy .J. Energy Conversion\&Management, (2009).

6. Kaempf J H, Robinson D.A simplified thermal model to support analysis of urban resource flows .J. Energy and Buildings, (2007).

7. Kalsi K, Elizondo M, Fuller J,et a1.Development and Validation of Aggregated Models for Thermostatic Controlled Loads with Demand
Response .C.// Hawaii International Conference on System Sciences. IEEE Computer Society, (2012).

8. Malhame R, Chong C Y.Electric load model synthesis by diffusion approximation of a high-order hybrid-state stochastic system .J. IEEE Transactions on Automatic Control, (2003).

9. Barrias F, Faria P, Vale Z.Consumption Management of Air Conditioning Devices for the Participation in Demand Response Programs .J. International Journal of Climatology, (2014).

10. Lu, Ning.An Evaluation of the HVAC Load Potential for Providing Load Balancing Service .J. IEEE Transactions on Smart Grid, (2012).

11. Nguyen $\mathrm{H} \mathrm{N}$ T, Zhang C, Zhang J.Dynamic Demand Control of Electric Vehicles to Support Power Grid with High Penetration Level of Renewable Energy .J. IEEE Transactions on Transportation Electrification, (2016). 